\title{
Effect of age and diet on hormone function
}

\section{By M. R. Turner, British Nutrition Foundation, Belgrave Square, London SWI}

The entire endocrine and nervous system is involved in the regulation of the intake and assimilation of nutrients. Among the hormones insulin has a particularly prominent role and, together with growth hormone and the somatomedins, will be considered particularly, but the concepts illustrated may be applicable to a wider range of hormones.

There are both quantitative and qualitative changes in endocrine function with age. For example, in the very young glucose tolerance - which is a measure of the net effect of insulin on the disappearance of glucose from the blood into target tissues-is very poor but increases with age until an adult level is reached. This has been shown in several species including man and is well documented for the dog (Heard \& Turner, 1967) and the rabbit (Turner, Bryant \& Carter, unpublished results). However, in the very young, insulin may have a more prominent effect on protein metabolism and growth than it does on energy metabolism-the requirement early in life is for growth whereas later in life the need for nutrient storage predominates.

Changes in glucose tolerance with age have been shown to result substantially from changes in the sensitivity of target tissues to the action of insulin (Heard \& Turner, 1967; Heard \& Henry, 1969; Turner, Bryant \& Carter, unpublished results), although changes in insulin secretion with age may also occur. However, because insulin secretion can be greater in the young when glucose tolerance is poor than it is in the adult when tolerance has improved, the importance of variations in tissue sensitivity to the hormone is emphasised. There is a close correlation between glucose tolerance and insulin sensitivity and this has been described clearly for the dog (Heard \& Henry, 1969; Heard, 1978) and the rabbit (Turner, Bryant \& Carter, unpublished results). The major effect of variations in insulin secretion will be seen when this is impaired, as in the diabetic.

Variations in diet are also capable of influencing endocrine function. Himsworth studied extensively the effect of short-term variations in diet in human subjects in whom he measured glucose tolerance and insulin sensitivity. He came to the conclusion that the only dietary factor affecting tolerance was carbohydrate, restriction of which resulted in insulin resistance and impairment of glucose tolerance (Himsworth, 1935). However, his conclusions are not valid for all dietary carbohydrates nor for long-term variations in dietary habits.

Increasing the dietary sucrose content results in impairment of glucose tolerance which may or may not be associated with changes in insulin secretion but, when this has been measured, is always associated with a reduction in insulin sensitivity. This has been shown in rats given diets containing sucrose providing up to $70 \%$ of 
the energy (Cohen \& Tertelbaum, r 964$)$ and is amply confirmed in rabbits given diets containing $32 \%$ of the energy as sucrose over a six month period (Turner, Bryant \& Carter, unpublished results). The work in rabbits also showed that although glucose tolerance was impaired by sucrose-rich diets consequent upon a reduction in insulin sensitivity, the age-related changes still occurred. Reintroduction of a low sucrose diet resulted in all cases in complete recovery over 2-3 months of both insulin sensitivity and glucose tolerance with no change in insulin secretion (Turner, Bryant \& Carter, unpublished results). These observations in rabbits are summarised in Figures $\mathbf{I}$ and 2.
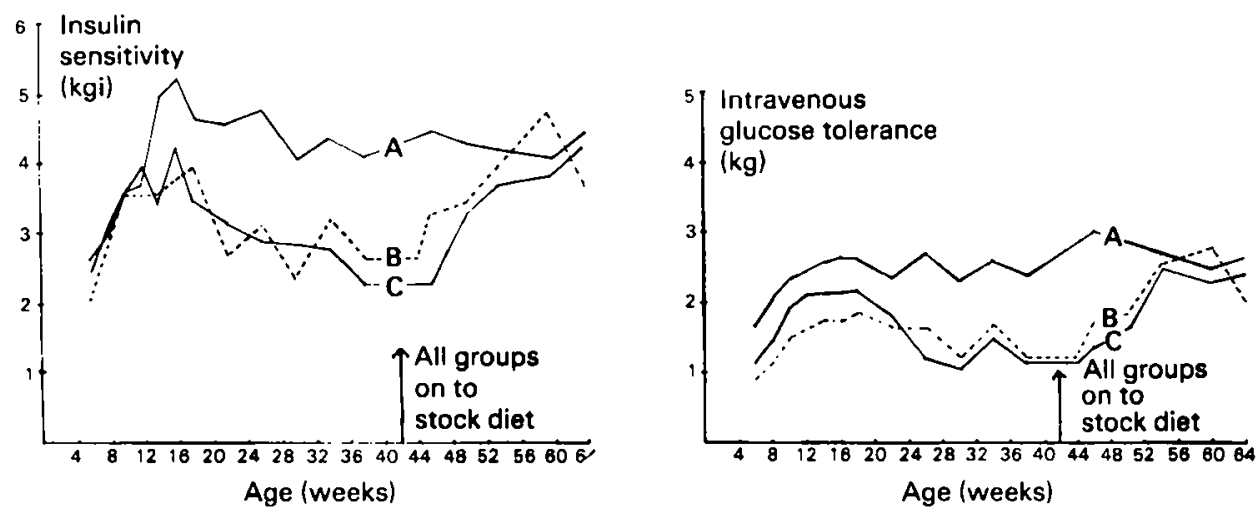

Figures I and 2. Intravenous glucose tolerance (kg) and insulin sensitivity (kg) in rabbits bred from mothers receiving a stock diet $(A)$, an experimental diet containing protein, $18 \%$ and sucrose, $8 \%$ of the energy (B), or protein, $12 \%$ and sucrose, $8 \%$ of the energy (C). From weaning offspring from group A continued to receive the stock diet, offspring from groups $\mathrm{B}$ and $\mathrm{C}$ received a diet containing protein, $18 \%$ and sucrose, $32 \%$ of the energy until 44 weeks of age after which all animals received the stock diet. (Turner, Bryant \& Carter, unpublished results).

The mechanism of the changes in insulin sensitivity associated with sucrose-rich diets is not understood. Insulin secretion in the rabbits given sucrose-rich diets was entirely normal for most of the experimental period, the exception being the first few weeks after weaning, and there was no evidence of excess adiposity, which is associated with insulin resistance in the obese in whom hyperinsulinaemia is also observed.

A long-term reduction in protein intake whether or not associated with reduction in total energy intake (protein-energy malnutrition-PEM) is also associated with impairment of glucose tolerance but in this instance there is also a reduction in insulin secretion. Insulin sensitivity has not been measured in human patients but in experimental PEM impairment of glucose tolerance is, again, largely a result of loss of insulin sensitivity although the impairment of insulin secretion also contributes. Other endocrine changes include high levels of plasma cortisol and growth hormone, the latter being associated with reduced secretory responses to suitable stimuli and failure of the plasma growth hormone to fall in the face of hyperglycaemia (reviewed by Turner 1972; Lunn et al. 1973).

The modern concept of hormonal influences on the growth process is that 
insulin and other insulin-like substances including the somatomedins play an important part. The somatomedins are released from the liver and kidney under stimulation by growth hormone and the modulating effects of other hormones. Growth hormone can therefore be regarded truly as a pituitary trophic hormone. Its direct metabolic action is the stimulation of lipolysis, a property that it shares with other pituitary trophic hormones such as ACTH and TSH whereas its growth promoting action is moderated by the somatomedins (Figure 3 ). The high levels of growth hormone in the malnourished therefore do not indicate increased growth promoting activity but increased lipolysis, a necessary function both between meals and when the diet is inadequate.

It has also been reported that in PEM there is a reduction in plasma concentration of somatomedins which is the logical adaptation to an adequate diet and there are also indications in PEM that an inhibitor of somatomedin action appears in the plasma (reviewed by Pimstone 1976). When PEM patients are reintroduced to normal feeding recovery of glucose tolerance and insulin secretion occurs and the growth hormone, somatomedin and cortisol levels return to normal over a period of time.

Thus, the diet-induced changes in endocrine function described appear to be more or less completely reversible.

The now classic observations of Widdowson and McCance (1963) have shown that when food restriction occurs sufficiently early in life-during the hyperplasia phase-there is permanent growth stunting whereas undernutrition later in life

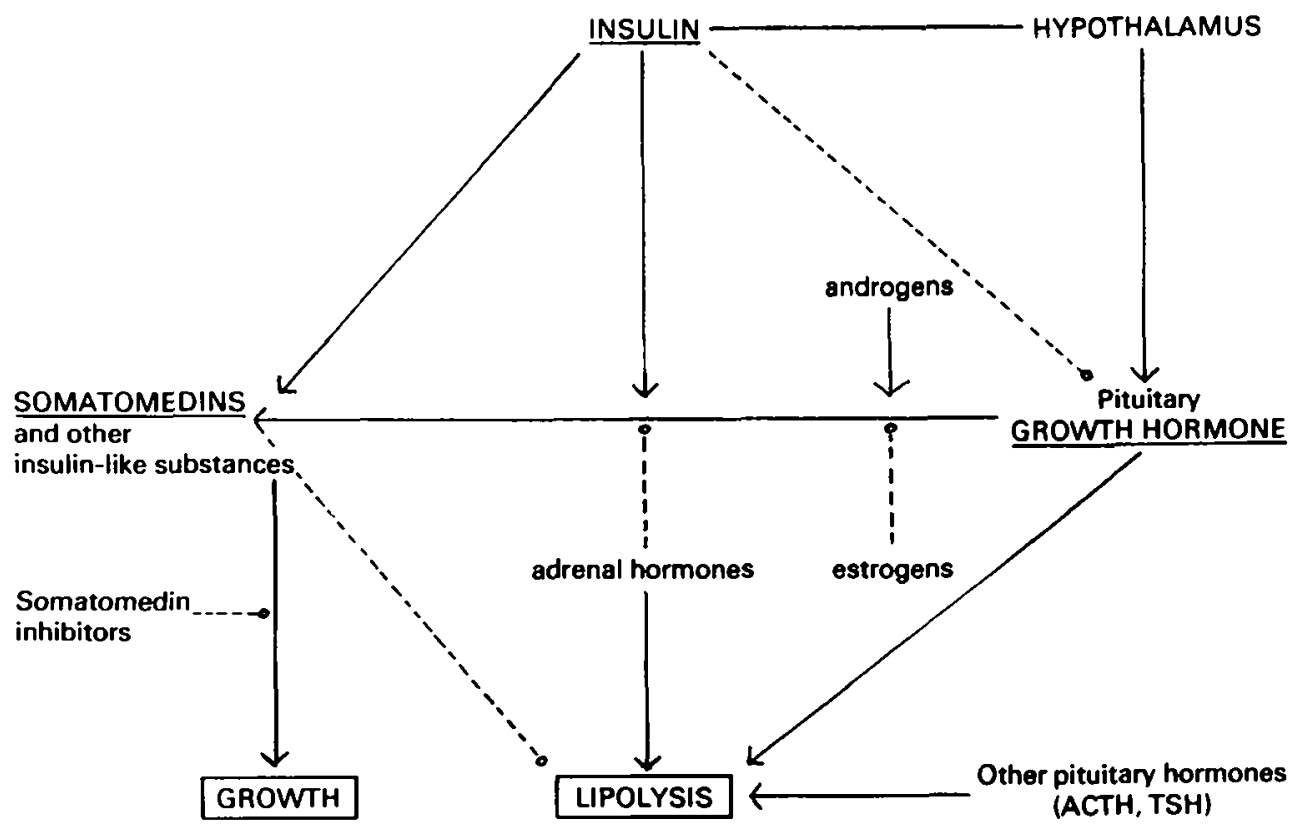

Figure 3. A scheme illustrating actions of growth hormone on lipolysis and growth: Stimulation, ( $\longrightarrow)$; inhibition, (- - -). 
during the hypertrophic phase of development leads to weight loss which is reversed when normal feeding is recommenced.

It is now well known that severe food restriction during gestation interferes with the normal development of the brain and associated with this there may be poor development of the pituitary (Stephan et al. 1971). However, experiments with severe dietary restrictions have limited practical value. An investigation was set up therefore to examine whether changes in the dietary protein and sucrose content of a type known to produce reversible changes in endocrine function in older animals would, when introduced very early in life, result in permanent changes in insulin function. Rabbits were given from mating diets moderately deficient in protein, but there was no reduction in energy intake. The sucrose content of the diets was also varied. The diets given to the reproducing females contained 9,12 or $18 \%$ of the energy as protein and 8 or $32 \%$ of the energy as sucrose. All diets contained $36 \%$ of the energy as fat. All offspring were weaned onto the high protein, high sucrose, high fat diet which was intended to simulate the type of diet typical of the UK. Comparisons were also made with rabbits reared and maintained throughout their lives on a stock diet (R. A. G. Christopher Hill Ltd., Poole, Dorset).

It was found that no offspring of mothers given the $9 \%$ protein diet survived to weaning, and survival of the offspring of all groups receiving the diets with the higher sucrose content was also very poor, for reasons which are not yet clear but which need further investigation (Turner, Bryant \& Carter, unpublished results).

Permanent growth stunting occurred in the offspring of mothers given the $12 \%$ protein diet and there was impairment in glucose tolerance and insulin sensitivity, no change due to diet in insulin secretion or blood lipids, and an exaggerated blood glucose increment after intravenous glucagon injection. However, very similar metabolic changes occurred in the control group of offspring of rabbits exposed to a similar dietary situation except that the mothers were given a diet with a higher protein content $(18 \%)$. In this group the offspring were not growth stunted. As both the $12 \%$ and $18 \%$ protein groups were receiving the same high sucrose diet from weaning and had similar defects in insulin function, the effects observed must be attributed to the increase in dietary sucrose post weaning. When all animals were put on the stock diet at 44 weeks of age complete recovery of glucose tolerance and insulin sensitivity occurred in both dietary groups.

We concluded, therefore, that the congenital moderate protein deficiency resulted in impairment of growth potential which was not related to insulin function and that high sucrose feeding from weaning resulted in impairment of glucose tolerance and insulin sensitivity, which was reversible. There was no evidence available from the limited number of animals that survived to weaning in the groups in which the mothers were given high sucrose diets whether or not early exposure to sucrose-rich diets had any effect on these particular metabolic variables, and whether or not the high death rate in this group was related to impairment of insulin function.

It is evident for insulin that the major factor determining net hormone function is variation in tissue sensitivity to the hormone, a concept that is familiar to those 
working on the problems of the obese. Indeed studies of the hormone:tissue interaction in vitro are now quite fashionable. For a long time, major emphasis has been placed on the secretory response to a 'challenge'. Perhaps the time has come to place more emphasis (as some workers are doing) on measurements of hormone concentration under normal physiological circumstances and, at the same time, pay much more attention to the functional implications of changes in the tissue responsiveness to hormones. This thought could be borne in mind not only by research workers but by those who modify growth, and plan to improve milk production, on the farm by manipulating the endocrine equilibrium and by doctors seeking the correct defects in the endocrine system of their patients.

In view of the apparent sensitivity of the endocrine system to dietary variations, a comment from an ancient Chinese source may be worth repeating (in free translation), '... a true doctor first finds out the cause of a disease and having found that out he tries to cure it first by food. If that fails, then he prescribes medicine...' (Sun Ssemaio-6th century A.D.).

\section{REFERENCES}

Cohen, A. M. \& Tertelbaum, A. (1964). Am. Y. Physiol. 206, 105.

Heard, C. R. C. (1978). In World Review of Nutrition and Dietetics, Vol. 30, pp. 107-147, [G. H. Bourne, editor]. Basel: Karger.

Heard, C. R. C. \& Henry, P. A. J. (1969). Clin. Sci. 37, 37.

Heard, C. R. C. \& Tumer, M. R. (1967). Diabetes 16, 96 .

Himsworth, H. P. (1935). Clin. Sci. 2, 67.

Lunn, P. G., Whitehead, R. G., Hay, R. W. \& Baker, B. A. (1973). Br. J. Nutr. 29, 399.

Pimstone, B. L. (1976). Clin. Endocr. 5, 79.

Stephan, J. K., Chow, B., Frohman, L. A. \& Chow, B. F. (1971). F. Nutr. ro1, 1453.

Sun Ssemaio (6th century A.D.) cited by Lin Yutang (1938). In The importance of living. London: Heinemann.

Turner, M. R. (1972). Proc. Nutr. Soc. 31, 205.

Widdowson, E. M. \& McCance, R. A. (1963). Proc. R. Soc. B. 158, 329. 